\title{
Uncertainty in Multiple Penetration Depth Fringing Electric Field Sensor Measurements
}

\author{
A. V. Mamishev, Member, IEEE, S. R. Cantrell, Y. Du, B. C. Lesieutre, and M. Zahn
}

\begin{abstract}
Measurement of multilayer material properties with fringing electric field dielectrometry sensors requires the processing of terminal admittance data from multiple electrode pairs. The choice of the algorithmic approach for data analysis is critical because of a high sensitivity to small measurement uncertainties. While individual channel uncertainties are usually within a reasonable instrumentation range, measurement of properties of multiple material layers fails unless special techniques are used to prevent numerical instabilities. This paper demonstrates the mechanism of uncertainty in a successive stair-step property estimation in which the estimates of one layer's properties are used to estimate the subsequent layer's properties. Three-wavelength measurements of dielectric permittivity and conductivity illustrate the problem in two-layer experimental setups. Limitations of the stair-step approach and possible future improvements are discussed.
\end{abstract}

Index Terms-Interdigital sensors, measurement uncertainty, multiple penetration depth.

\section{INTRODUCTION}

I NTERDIGITAL sensors are widely used for nondestructive measurement of material properties. Most commonly, interdigital electrodes are encountered in surface acoustic wave (SAW) devices [1], but they are also used for fringing electric field measurements, capacitive measurements, in MEMS, and in chemical and biological applications [2]. The focus of this work is on multiple penetration depth fringing electric field measurements with a three-wavelength interdigital sensor. Work in this area had been conducted as early as in the 1960s [3]. Since then, a number of theoretical and experimental investigations have contributed to different aspects of this technology, including electric field modeling [4], [5], industrial and scientific applications [6], [7], design optimization [8], and parameter estimation [9].

The measurement of dielectric properties of insulating and semi-insulating materials provides indirect information about other physical properties of these materials, for example, moisture concentration, density, porosity, structural integrity,

Manuscript received February 15, 2002; revised October 3, 2002. This work was supported by the Electric Power Research Institute, under Grant WO 8619-01, managed by S. Lindgren, the National Science Foundation under Grant ECS-9523128, the Center of Processing Analytical Chemistry, and NSF Award ECS-0093716. Y. Du was supported by the APPA DEED Scholarship. A. V. Mamishev was supported by the APPA DEED Scholarship and the Link Foundation Fellowship.

A. V. Mamishev and S. R. Cantrell are with the Department of Electrical Engineering, University of Washington, Seattle, WA 98195 USA (e-mail: mamishev@ee.washington.edu).

Y. Du is with the Underwriters Laboratories, Inc.

B. C. Lesieutre is with Cornell University, Ithaca, NY 14853 USA.

M. Zahn is with the Massachusetts Institute of Technology, Cambridge, MA 02139 USA.

Digital Object Identifier 10.1109/TIM.2002.808013

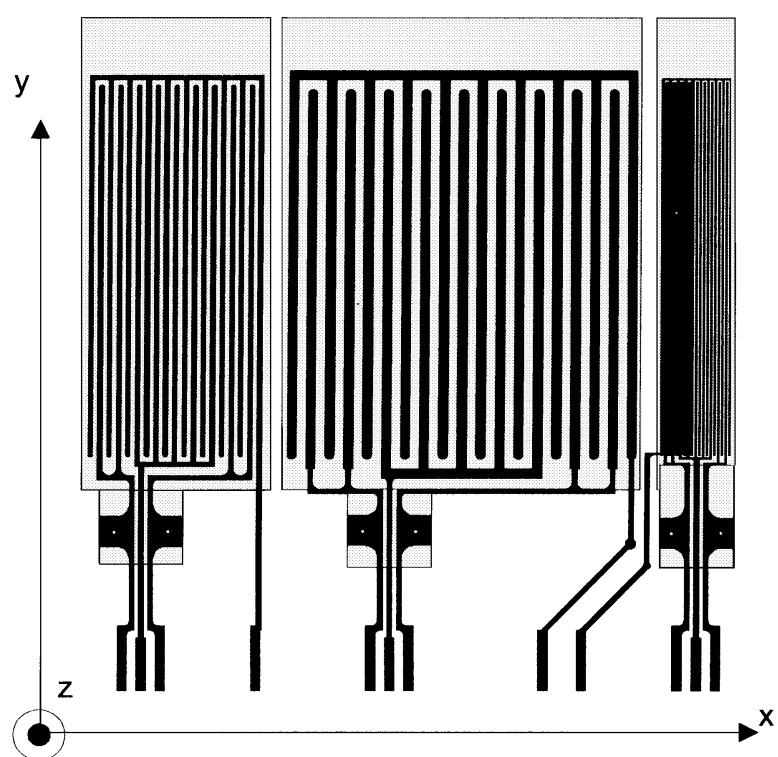

Fig. 1. Three-wavelength interdigital sensor with spatial periodicities of 2.5 $\mathrm{mm}, 5.0 \mathrm{~mm}$, and $1.0 \mathrm{~mm}$.

concentration of impurities, thickness of laminations, etc. In the simplest case, dielectric properties of homogeneous samples can be determined and related to other variables of interest through mapping experiments by determining the relationship between the dielectric properties and other variables in parallel plate setups. A more sophisticated and useful task is to determine properties of different regions in a nonhomogeneous sample. In this case, a variety of dynamic processes can be studied nondestructively as well.

The multiple penetration depth fringing electric field dielectrometry sensor used in this study is shown in Fig. 1 [8]. It consists of three sets of topologically identical interdigital electrodes etched on a common flexible Teflon substrate. The gray shaded area indicates guard backplanes on the reverse side of the substrate. Measurement of material properties at different depths from the surface is achieved through variation of spatial periodicity of interdigital electrode pairs. The larger the distance between the electrode centerlines, the deeper is the effective penetration depth. Ideally, appropriate processing of signals from all pairs should provide an adequate picture of material property distributions that only vary with the $z$ coordinate and do not change with $x$ or $y$ coordinates across the sensor head. The transadmittance between electrode pairs is measured by driving one electrode with a known sinusoidal voltage signal and measuring the terminal current on the other electrode. In our normal practice, the frequency of this signal varies between $0.005 \mathrm{~Hz}$ and $10 \mathrm{kHz}$. Several parameter estimation algorithms 


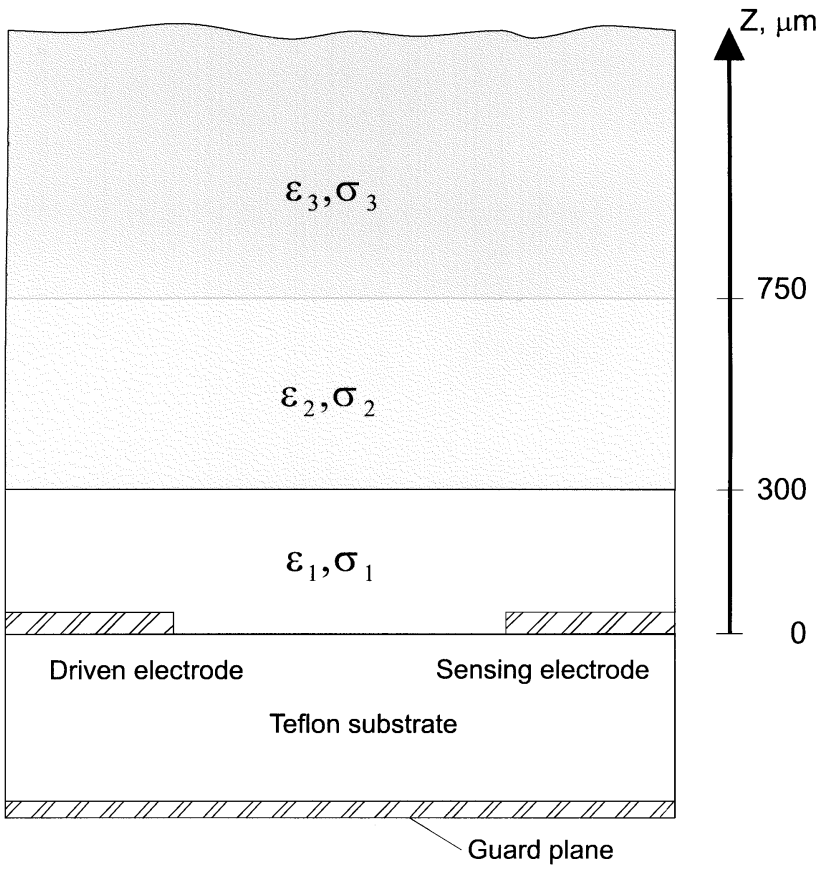

Fig. 2. Three layers with unknown permittivity and conductivity can be characterized by a half-wavelength model.

have been constructed to relate transadmittance values to dielectric permittivity and conductivity of a material layer under test at different depths in a multilayer system.

This sensor has been used in several preliminary studies aimed at measurements of moisture diffusion through electric insulation of power transformers [10], [11]. Since no other experimental technique is currently available for verification of measured distributions of moisture concentration, the estimation of sensitivity and potential measurement uncertainty becomes particularly important. Many theoretical and experimental approaches are available for sensitivity analysis. The goal of this paper is to present results of simulations and measurements that are directly applicable to the stair-step inversion algorithm. In this algorithm, dielectric properties close to the sensor are estimated first, and then used to estimate properties of consecutive layers [4], [7].

This paper treats issues related to sensitivity and accuracy of parameter estimations from interdigital dielectrometry measurements. The influence of small disturbance factors of nondestructive measurements grows dramatically with the number of unknown variables that characterize materials under test. In the framework of inverse problem theory it means that the problem of material characterization is ill-conditioned, because a high variation in output parameters occurs due to the small variation of input parameters. To justify this claim, a numerical example of uncertainty and example equations are presented here and then followed by measurement verification. The results of measurements also illustrate the potential of this technological approach and indicate its current limitations.

\section{UNCERTAINTY PROPAGATION}

\section{A. General Procedure}

Finite element method computer simulations are used to estimate uncertainty propagation for a specific case of perfectly insulating three-layer media. The results of these simulations show how a small measurement uncertainty may lead to completely incorrect estimation of material properties. The limitations of the stair-step inverse problem approach arise mostly from this phenomena.

Consider the three-layer system shown in Fig. 2. Ideally, using a three-wavelength sensor with penetration depths comparable to the interface positions of the three layers, one should be able to determine the dielectric properties of each layer. For simplicity, let us consider a nonconducting case, so that $\sigma_{1}, \sigma_{2}$, and $\sigma_{3}$ are equal to zero. Then, the three permittivities to be determined from the three-wavelength measurements are $\varepsilon_{1}, \varepsilon_{2}$, and $\varepsilon_{3}$. For the purpose of this numerical example, the distance between the top of the sensor electrodes and the interfacial boundaries of the corresponding layers is chosen to be 0.3 of the spatial wavelength. So, the thickness of the first layer is $300 \mu \mathrm{m}$, the thickness of the second layer is $750-300=450 \mu \mathrm{m}$, and the thickness of the third layer is assumed to be infinite. To start, we assume all three layers have identical relative permittivity, $\varepsilon_{1}=\varepsilon_{2}=\varepsilon_{3}=\varepsilon$, so that the transcapacitance of each wavelength is only a function of $\varepsilon$. For a particular operating point we use Teflon with $\varepsilon=2.1$.

Whenever an infinite homogeneous layer is used in the simulations below, the dielectric permittivity of the layer or layers close to the sensor determines and is equal to the dielectric permittivity of the upper layers. The infinite homogeneous layer always starts at the penetration depth $(0.3 \lambda)$ of the simulated wavelength. For clarity, these assumptions are stated at each figure caption.

\section{B. General Uncertainty}

Along with the simulated plots, general uncertainty is used in example equations. The general uncertainty equation is

$$
U_{r}^{2}=\sum_{i=1}^{J}\left(\frac{\partial r}{\partial X_{i}} U_{X_{i}}\right)^{2}
$$

where $r$ is the variable with the uncertainty that varies depending on the $J$ independent variables $X_{i}$

$$
r=r\left(X_{1}, X_{2}, \ldots, X_{J}\right) \text {. }
$$

$U_{r}$ is the uncertainty in the variable $r$, and the variables $U_{X_{i}}$ represent the independent uncertainties in the variables $X_{i}[12]$. Uncertainties are typically measured in a region of $95 \%$ confidence. For example, if a variable $X$ has a region of $95 \%$ confidence that spans $\pm 3 \%$ of the nominal value, then $U_{X} / X$ is $2 \cdot 3 \%=6 \%$. If the uncertainty, $U_{X}$, remains constant, notice that the percentage of uncertainty shrinks as the nominal value $X$ increases. In the case of the plots, $U_{X}$ is the size of the region of uncertainty (the absolute difference between the high and low bounding values), and $X$ is the nominal value.

A convenient way to calculate uncertainties is as the ratio $U_{r} / r$, which is the format that this paper uses. Here the equation is reorganized for clarity

$$
\left(\frac{U_{r}}{r}\right)^{2}=\sum_{i=1}^{J}\left(\frac{1}{r} \frac{\partial r}{\partial X_{i}} U_{X_{i}}\right)^{2} .
$$




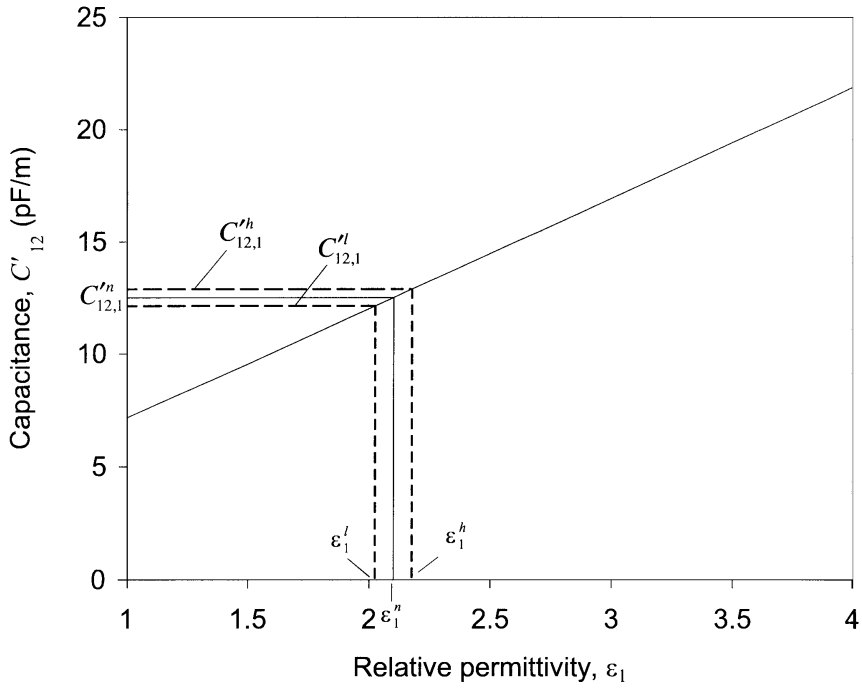

Fig. 3. Simulated function curve of the sensor with 1.0-mm wavelength and a capacitance uncertainty of $6 \%$; the uncertainty shown for $\varepsilon_{1}$ is approximately $\left|\varepsilon_{1}^{h}-\varepsilon_{1}^{l}\right| / \varepsilon_{1}^{n}=7.2 \%$.

\section{Nomenclature}

In this paper, relative dielectric permittivities are labeled with a subscript $i$ as in $\varepsilon_{i}$ where $i$ is an integer that represents the layer. The numbering starts with the layer closest to the sensor, $i=1$, and increases sequentially for more remote layers. The combined layers are described with the permittivity subscripts of each of the layers; for example, the combined first and second layers measured by the 2.5-mm wavelength are described with the permittivity $\varepsilon_{12}$, where $\varepsilon_{12}=\varepsilon_{1}=\varepsilon_{2}$. Capacitances are labeled with two subscripts, $a$ and $b$ as in $C_{a, b}^{\prime}$; the prime shows that the value is in units of farads per meter instead of just farads. The subscript $a$ specifies the two nodes that the capacitance spans in the circuit (for example, in this paper $a=12$ because the material is assumed to be between nodes 1 , the driven electrode, and 2, the sensing electrode); $b$ specifies the sensor wavelength being used. In this case, 1, 2, and 3 correspond with the $1.0 \mathrm{~mm}, 2.5 \mathrm{~mm}$, and $5.0 \mathrm{~mm}$ spatial wavelengths, respectively. The variables can also have an extra superscript: $n$ for nominal ( $n$ is left off in equations for simplicity), $h$ for the highest value of uncertainty, or $l$ for the lowest value of uncertainty. For example, $C_{12,2}^{\prime h}$ would be the high value of uncertainty seen between the nodes 1 (the driven electrode) and 2 (the sensing electrode) from the second sensor ( $2.5 \mathrm{~mm}$ separation).

\section{One-Layer Parameter Estimation}

Fig. 3 shows the transcapacitance for the $1 \mathrm{~mm}$ wavelength computed on a per meter length basis for an infinitely thick layer of relative permittivity $\varepsilon=\varepsilon_{1}$. It is nearly linear, and the $\pm 3 \%$ uncertainty bounds in the measurement of interelectrode capacitance leads to $\pm 3.8 \%$ uncertainty in the estimated value of relative dielectric permittivity around $\varepsilon_{1}=2.1$. The $\pm 3 \%$ uncertainty bound is an assumption based on a statistical description of our extensive experimental data. It combines known and unknown disturbance factors, including electronic equipment noise, round-off due to A/D conversion, manufacturing tolerances of the sensor itself, accuracy of known geometric parameters, effects of the finite length and width of the sensor head

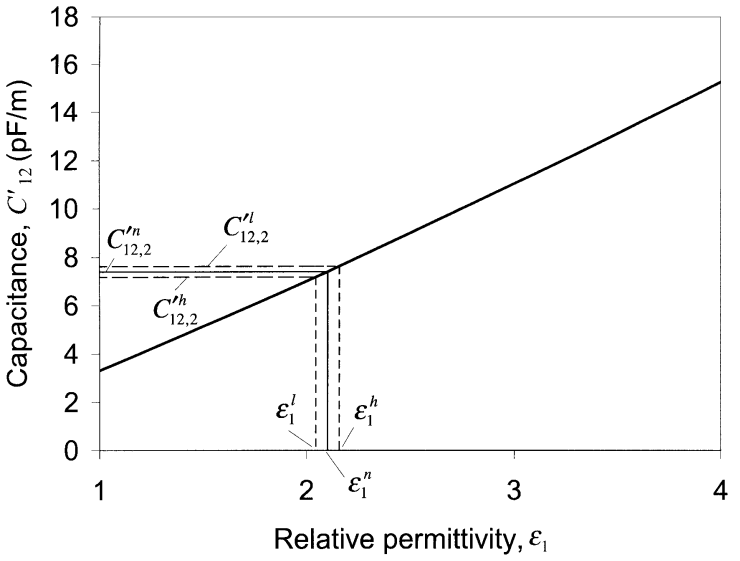

Fig. 4. Simulated function curve of the sensor with $2.5-\mathrm{mm}$ wavelength and a capacitance uncertainty of $6 \%$; the uncertainty shown for $\varepsilon_{1}$ is approximately $\left|\varepsilon_{1}^{h}-\varepsilon_{1}^{l}\right| / \varepsilon_{1}^{n}=5.4 \%$.

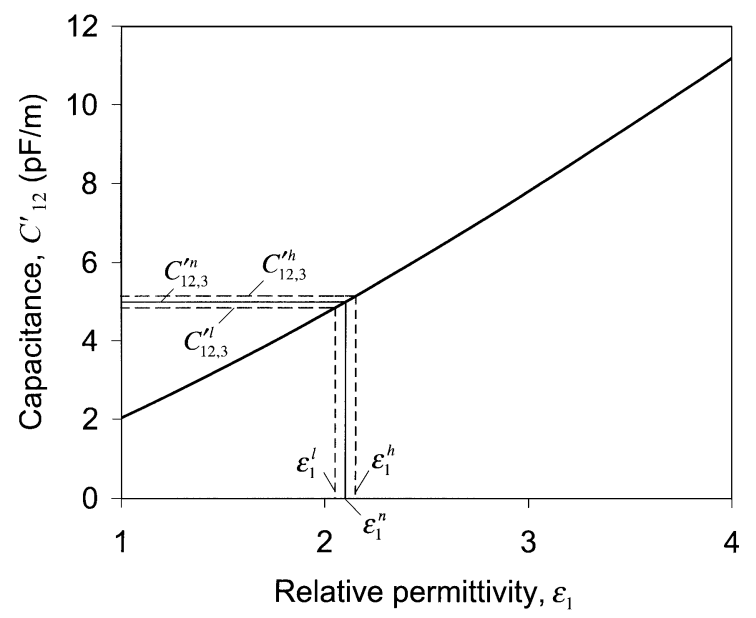

Fig. 5. Simulated function curve of the sensor with 5.0-mm wavelength and a capacitance uncertainty of $6 \%$; the uncertainty shown for $\varepsilon_{1}$ is approximately $\left|\varepsilon_{1}^{h}-\varepsilon_{1}^{l}\right| / \varepsilon_{1}^{n}=4.8 \%$.

not included in the two-dimensional half-wavelength cell model shown in Fig. 2, and others. Fig. 4 shows the transcapacitance for the $2.5 \mathrm{~mm}$ wavelength, again, assuming an infinitely thick layer of material with $\varepsilon=\varepsilon_{12}$. The $\pm 3 \%$ uncertainty in the estimation of the interelectrode capacitance $C_{12}$ leads to a $\pm 2.7 \%$ uncertainty in the estimated value of $\varepsilon$. Fig. 5 shows results of the same type of calculation for the $5 \mathrm{~mm}$ wavelength, with the uncertainty in the estimated value of $\varepsilon=\varepsilon_{123}$ being equal to $\pm 2.4 \%$. Given the assumed $\pm 3 \%$ measurement uncertainty, the accuracy of material property estimation for a single layer of infinite extent is acceptable in all the above cases. However, as will be shown, as the number of unknowns grows in a multilayer system, the uncertainty of measurements very quickly becomes unreasonably high.

\section{E. Example Equations for One Layer}

The relationship between capacitance and permittivity is not linear, which is characteristic of fringing field capacitors. Because electric field lines change shape with the change of material properties, the ratio of energy amounts stored in the substrate and in the material under test varies with the capacitance. 
TABLE I

VALUES $a_{1}, a_{2}, a_{3}$, AND $b$ USED IN THE EQUATIONS THAT CORRESPOND WITH THE GIVEN FIGURES

\begin{tabular}{l|c|c|c|c}
\hline Figure & $a_{1}(\mathrm{~m} / \mathrm{pF})$ & $a_{2}(\mathrm{~m} / \mathrm{pF})$ & $a_{3}(\mathrm{~m} / \mathrm{pF})$ & $\mathrm{b}$ \\
\hline 3 & 0.204 & - & - & -0.446 \\
\hline 4 & 0.232 & - & - & 0.383 \\
\hline 5 & 0.321 & - & - & 0.495 \\
\hline 6 & -0.613 & 1.059 & - & 1.910 \\
\hline 7 & -0.181 & 0.628 & - & 1.225 \\
\hline 8 & -2.393 & -0.010 & 1.399 & 25.051 \\
\hline
\end{tabular}

For uncertainty calculations, each of these functions is approximated with a linear equation

$$
\varepsilon_{1}=a C_{12,1}^{\prime}+b
$$

where $a$ is the slope and $b$ is the $C^{\prime}$ intercept for a line approximating the nonlinear function around the point $\left(\varepsilon_{1}, C_{12,1}^{\prime n}\right)$. The values used in this and the preceding equations are given in Tables I and II. The following equations are then generated for the sensor with a spacing of $1.0 \mathrm{~mm}$ using the general uncertainty equations

$$
\begin{aligned}
& \left(\frac{U_{\varepsilon_{1}}}{\varepsilon_{1}}\right)^{2}=\left[\frac{U_{C_{12,1}^{\prime}}}{\varepsilon_{1}}\left(\frac{\partial \varepsilon_{1}}{\partial C_{12,1}^{\prime}}\right)\right]^{2} \\
& \left(\frac{U_{\varepsilon_{1}}}{\varepsilon_{1}}\right)^{2}=\left[\frac{a U_{C_{12,1}^{\prime}}}{a C_{12,1}^{\prime}+b}\right]^{2} .
\end{aligned}
$$

Using the plots, the following values are estimated:

$$
U_{C_{12,1}^{\prime n}} / C_{12,1}^{\prime n} \cong 6.0 \%, \quad U_{\varepsilon_{1}} / \varepsilon_{1} \cong 7.2 \% \text {. }
$$

Thus, a capacitance uncertainty of $6.0 \%$ produces approximately $7.2 \%$ output uncertainty in the permittivity. Following these same steps with the other two sensor wavelengths produces

$$
U_{C_{12,2}^{\prime n}} / C_{12,2}^{\prime n} \cong 6.0 \%, \quad U_{\varepsilon_{2}} / \varepsilon_{2} \cong 4.9 \%
$$

for the $2.5 \mathrm{~mm}$ separation and

$$
U_{C_{12,3}^{\prime \prime}} / C_{12,3}^{\prime n} \cong 6.0 \%, \quad U_{\varepsilon_{3}} / \varepsilon_{3} \cong 4.6 \%
$$

for the 5-mm separation.

Notice how the uncertainty goes down as the separation increases between the electrodes. This is because the increase in separation spreads more of the electric field through the material under test, which increases the sensor's sensitivity. Also notice that these uncertainty values do not match the simulated ones shown in the figures. This is because the estimated equations do not match the simulated data perfectly. The equations assume a perfectly linear dependence and approximate the data in a least squares sense. While it is possible to achieve a closer match by using a tangential line at the point of measurement instead of least squares fit, it would serve no practical purpose.

\section{F. Two-Layer Parameter Estimation}

Suppose that we want to measure properties of a two-layer material under test and consider two cases. In the first case, the results of measurements with two neighboring wavelengths, $1 \mathrm{~mm}$ and $2.5 \mathrm{~mm}$ are used to estimate material properties. In the second case, the two spatial wavelengths are more dissimilar ( $1 \mathrm{~mm}$ versus $5 \mathrm{~mm}$ ). The results of simulations for the first case are shown in Fig. 6. The top and bottom curves in this figure represent the extreme cases of the relative dielectric permittivity of the first layer ( $\varepsilon_{1}^{l}$ and $\varepsilon_{1}^{h}$ ) estimated based on the measurements with a $1 \mathrm{~mm}$ wavelength, shown in Fig. 3. The middle curve is the capacitance of the $2.5 \mathrm{~mm}$ wavelength channel computed assuming the correct dielectric permittivity $\left(\varepsilon_{1}^{n}=2.1\right)$ of the first layer. The dashed lines indicate the maximum uncertainty brackets assuming that the uncertainty in the measured values of capacitance of the $2.5 \mathrm{~mm}$ wavelength channel due to a combination of all disturbance factors does not exceed $\pm 3 \%$. One can see immediately that the uncertainty brackets for the relative dielectric permittivity of the second layer, $\varepsilon_{2}$, are unacceptably high, from $-22 \%$ to $+29 \%$ of the nominal value $\varepsilon_{2}^{n}=2.1$ $\left(\varepsilon_{2}^{l}=1.63, \varepsilon_{2}^{h}=2.70\right)$.

In the second case with the $1 \mathrm{~mm}$ and $5 \mathrm{~mm}$ wavelengths, shown in Fig. 7, the error brackets of the relative dielectric permittivity estimation are significantly more narrow, about $\pm 8 \%$ of the nominal value $\varepsilon_{2}^{n}=2.1\left(\varepsilon_{2}^{l}=1.93, \varepsilon_{2}^{h}=2.27\right)$.

\section{G. Example Equations for Two Layers}

Notice that the functional dependence of capacitance on permittivity is no longer a line, but rather a region, with the top boundary based on the $\varepsilon_{1}^{l}$ and the bottom boundary based on $\varepsilon_{1}^{h}$. Since the calculated permittivity depends on measurements from both sensor elements instead of just one, the following equation is the approximation used for the function shown in Fig. 6

$$
\varepsilon_{2}=a_{1} C_{12,1}^{\prime}+a_{2} C_{12,2}^{\prime}+b .
$$

The general uncertainty equation is then used to calculate the uncertainty

$$
\begin{aligned}
\left(\frac{U_{\varepsilon_{2}}}{\varepsilon_{2}}\right)^{2}= & {\left[\frac{U_{C_{12,1}^{\prime}}}{\varepsilon_{2}}\left(\frac{\partial \varepsilon_{2}}{\partial C_{12,1}^{\prime}}\right)\right]^{2}+\left[\frac{U_{C_{12,2}^{\prime}}}{\varepsilon_{2}}\left(\frac{\partial \varepsilon_{2}}{\partial C_{12,2}^{\prime}}\right)\right]_{(8)}^{2} } \\
\left(\frac{U_{\varepsilon_{2}}}{\varepsilon_{2}}\right)^{2}= & {\left[\frac{a_{1} U_{C_{12,1}^{\prime}}}{a_{1} C_{12,1}^{\prime}+a_{2} C_{12,2}^{\prime}+b}\right]^{2} } \\
& +\left[\frac{a_{2} U_{C_{12,2}^{\prime}}^{\prime}}{a_{1} C_{12,1}^{\prime}+a_{2} C_{12,2}^{\prime}+b}\right]^{2}
\end{aligned}
$$

The uncertainty values for the Fig. 6 case are

$$
\frac{U_{C_{12,1}^{\prime n}}}{C_{12,1}^{\prime n}} \cong 6.00 \%, \quad \frac{U_{C_{12,2}^{\prime n}}^{\prime n}}{C_{12,2}^{\prime n}} \cong 6.00 \%, \quad \frac{U_{\varepsilon_{2}}}{\varepsilon_{2}} \cong 31.32 \%
$$

and the values for the Fig. 7 case are:

$$
\frac{U_{C_{12,1}^{\prime n}}}{C_{12,1}^{\prime n}} \cong 6.00 \%, \quad \frac{U_{C_{12,3}^{\prime n}}}{C_{12,3}^{\prime n}} \cong 6.00 \%, \quad \frac{U_{\varepsilon_{2}}}{\varepsilon_{2}} \cong 11.06 \% .
$$

\section{H. Three-Layer Parameter Estimation}

A three-layer parameter estimation using a straightforward stair-step algorithm results in very large uncertainty brackets. Fig. 8 shows the uncertainty brackets for the relative dielectric permittivity of the third layer, $\varepsilon_{3}$, computed using previ- 
TABLE II

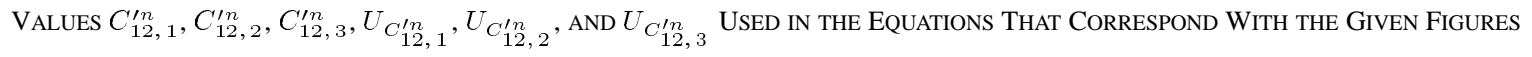

\begin{tabular}{l|c|c|c|c|c|c}
\hline Figure & $C_{12,1}^{\prime n}(\mathrm{~m} / \mathrm{pF})$ & $C_{12,2}^{\prime n}(\mathrm{~m} / \mathrm{pF})$ & $C_{12,3}^{\prime n}(\mathrm{~m} / \mathrm{pF})$ & $U_{C_{12,1}^{\prime n}}(\mathrm{~m} / \mathrm{pF})$ & $U_{C_{12,2}^{\prime n}}(\mathrm{~m} / \mathrm{pF})$ & $U_{C_{12,3}^{\prime n}}(\mathrm{~m} / \mathrm{pF})$ \\
\hline 3 & 12.481 & - & - & 0.749 & - & - \\
\hline 4 & - & 7.400 & - & - & 0.444 & - \\
\hline 5 & - & - & 5.000 & - & - & 0.300 \\
\hline 6 & 12.481 & 7.400 & - & 0.749 & 0.444 & - \\
\hline 7 & 12.481 & - & 5.000 & 0.749 & - & 0.300 \\
\hline 8 & 12.481 & 7.400 & 5.000 & 0.749 & 0.444 & 0.300 \\
\hline
\end{tabular}

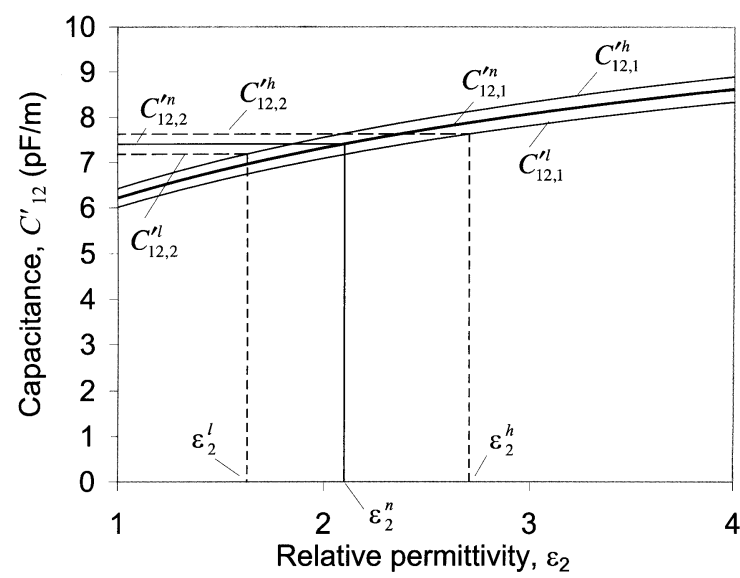

Fig. 6. Simulated function curve of the 2.5-mm sensor when determining $\varepsilon_{2}$ for the second layer. We use the three $\varepsilon_{1}$ values found with the $1.0-\mathrm{mm}$ wavelength as seen in Fig. 3. The capacitance uncertainty for both capacitive measurements is $6 \%$; the uncertainty shown for $\varepsilon_{2}^{n}$ is approximately $\left|\varepsilon_{2}^{h}-\varepsilon_{2}^{l}\right| / \varepsilon_{2}^{n}=51 \%$ [error brackets of the relative dielectric permittivity around the theoretical value $\varepsilon_{2}=2.1$ (with $\varepsilon_{2}=\varepsilon_{3}$ ) of the second layer calculated assuming a $\pm 3 \%$ error of the $2.5 \mathrm{~mm}$ wavelength interelectrode capacitance per meter length $C_{12}^{\prime}$ due to a combined influence of all disturbance factors and measurement noise for first layer permittivity values $\varepsilon_{1}^{l}, \varepsilon_{1}^{n}$, and $\varepsilon_{1}^{h}$ from Fig. 3].

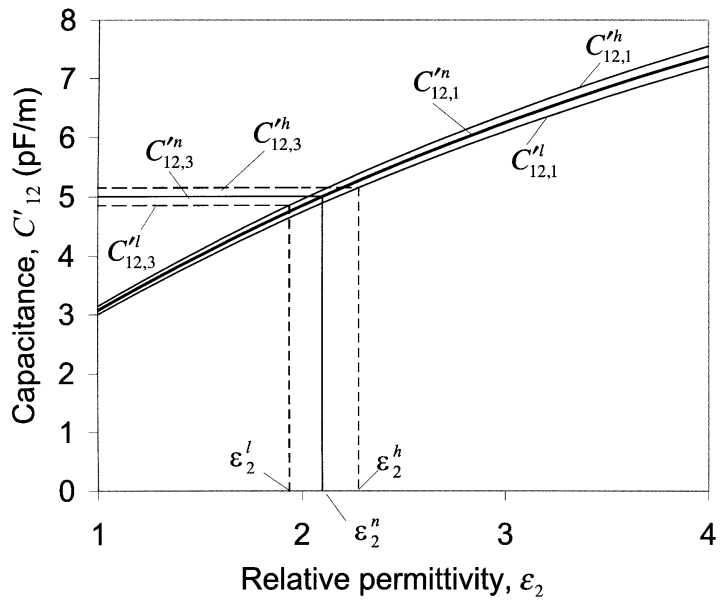

Fig. 7. Simulated function curve of the 5.0-mm sensor when determining $\varepsilon_{2}$ for the second layer. We use the three $\varepsilon_{1}$ values found with the $1.0-\mathrm{mm}$ wavelength as seen in Fig. 3. The capacitance uncertainty for both capacitive measurements is $6 \%$; the uncertainty shown for $\varepsilon_{2}$ is approximately $\left|\varepsilon_{2}^{h}-\varepsilon_{2}^{l}\right| / \varepsilon_{2}^{n}=16 \%$ [error brackets of the relative dielectric permittivity (with $\varepsilon_{2}=\varepsilon_{3}$ ) of the second and third layers calculated assuming a $\pm 3 \%$ error of the $5 \mathrm{~mm}$ wavelength interelectrode capacitance per meter length $C_{12}^{\prime}$ due to a combined influence of all disturbance factors and measurement noise for first layer permittivity values $\varepsilon_{1}^{l}, \varepsilon_{1}^{n}$, and $\varepsilon_{1}^{h}$ from Fig. 3].

ously estimated values of dielectric permittivities of the first and second layers, $\varepsilon_{1}$ and $\varepsilon_{2}$, respectively, found in Figs. 3 and 6 . Since for this electrode geometry the interelectrode capaci-

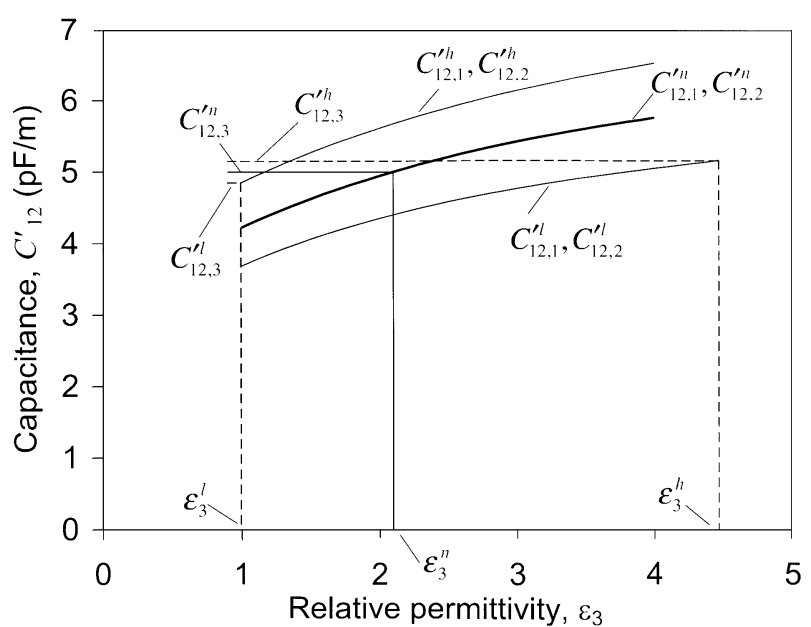

Fig. 8. Simulated function curve of the 5.0-mm sensor when determining $\varepsilon_{3}$ for the third layer. We use the three $\varepsilon_{1}$ values found with the 1.0 -mm and $2.5-\mathrm{mm}$ wavelengths, Figs. 3 and 6 respectively. The capacitance uncertainty is $6 \%$; the uncertainty shown for $\varepsilon_{3}$ is approximately $\left|\varepsilon_{3}^{h}-\varepsilon_{3}^{l}\right| / \varepsilon_{3}^{n}=166 \%$ [error brackets of the relative dielectric permittivity of the third layer calculated assuming a $\pm 3 \%$ error of the 5-mm wavelength interelectrode capacitance per meter length $C_{12}^{\prime}$ due to a combined influence of all disturbance factors and measurement noise for first and second layer permittivity values $\left(\varepsilon_{1}^{l}, \varepsilon_{2}^{l}\right),\left(\varepsilon_{1}^{n}\right.$, $\left.\varepsilon_{2}^{n}\right)$, and $\left(\varepsilon_{1}^{h}, \varepsilon_{2}^{h}\right)$ from Figs. 3 and 6$]$.

tance grows with the individual dielectric permittivities of each layer, the curves of the extreme cases use a combination of both highest $\left(\varepsilon_{1}^{h}, \varepsilon_{2}^{h}\right)$ or both lowest $\left(\varepsilon_{1}^{l}, \varepsilon_{2}^{l}\right)$ dielectric permittivities. This $\pm 3 \%$ uncertainty of the measured capacitance of the $5 \mathrm{~mm}$ wavelength channel results in a $-52 \%$ and $+114 \%$ uncertainty in the value of third layer permittivity around $\varepsilon_{3}^{n}=2.1$ $\left(\varepsilon_{3}^{l}=1.0, \varepsilon_{3}^{h}=4.45\right)$.

\section{Example Equations for Three Layers}

Since both $\varepsilon_{1}$ and $\varepsilon_{2}$ are used with the $5.0 \mathrm{~mm}$ sensor to generate $\varepsilon_{3}$, all three capacitance measurements are needed in our linear approximation of this curve

$$
\varepsilon_{3}=a_{1} C_{12,1}^{\prime}+a_{2} C_{12,2}^{\prime}+a_{3} C_{12,3}^{\prime}+b .
$$

Notice that the nonlinear effects are becoming large enough to cause asymmetry of high and low estimates with respect to the nominal values.

The basic uncertainty equation for this case is

$$
\begin{gathered}
\left(\frac{U_{\varepsilon_{3}}}{\varepsilon_{3}}\right)^{2}=\left[\frac{U_{C_{12,1}^{\prime}}}{\varepsilon_{3}}\left(\frac{\partial \varepsilon_{3}}{\partial C_{12,1}^{\prime}}\right)\right]^{2}+\left[\frac{U_{C_{12,2}^{\prime}}}{\varepsilon_{3}}\left(\frac{\partial \varepsilon_{3}}{\partial C_{12,2}^{\prime}}\right)\right]^{2} \\
+\left[\frac{U_{C_{12,3}^{\prime}}}{\varepsilon_{3}}\left(\frac{\partial \varepsilon_{3}}{\partial C_{12,3}^{\prime}}\right)\right]^{2}
\end{gathered}
$$


TABLE III

Two Layer Dielectrometry Measurements Using the GeOMEtry in Fig. 9 With Air OR CoRn OIL as Layer 1 and Air, CoRn OIL, LeXan, or Teflon

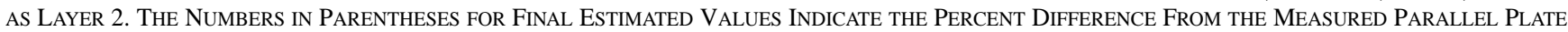

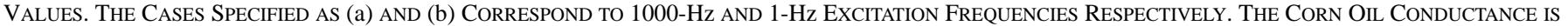
NOT ESTIMATED AT $1000 \mathrm{~Hz}$ (MARKed With “-”) WhEN the SENSITIVITY OF THE MEASUREMENT IS KNOWN to BE INSUFFICIENT

\begin{tabular}{|c|c|c|c|c|c|c|c|c|}
\hline \multicolumn{9}{|c|}{ Diclectric properties measured with guarded parallel-plate capacitor at $1 \mathrm{~Hz}$ and $1000 \mathrm{~Hz}$} \\
\hline & Air & \multicolumn{2}{|c|}{ Corn Oil } & Air/Lexan & \multicolumn{2}{|c|}{ Corn Oil/Lexan } & \multicolumn{2}{|c|}{ Corn Oil/Teflon } \\
\hline & Case 1a & Case 2a & Case $2 \mathrm{~b}$ & Case 3a & Case $4 \mathrm{a}$ & Case $4 \mathrm{~b}$ & Case 5 a & Case $5 b$ \\
\hline$f(\mathrm{~Hz})$ & 1000 & 1000 & 1 & 1000 & 1000 & 1 & 1000 & $\mathrm{I}$ \\
\hline$\varepsilon_{1}^{e}$ & 1.0 & 3.13 & 3.16 & 1.0 & 3.13 & 3.16 & 3.13 & 3.16 \\
\hline$\sigma_{1}^{e}(\mathrm{pS} / \mathrm{m})$ & 0 & - & 29.18 & 0 & - & 29.18 & - & 29.18 \\
\hline$\varepsilon_{2}^{e}$ & 1.0 & 3.13 & 3.16 & 3.01 & 3.01 & 3.01 & 2.1 & 2.1 \\
\hline \multicolumn{9}{|c|}{ Measured $C_{12}$ and $G_{12}$ for each of three wavclengths } \\
\hline$C_{12}(\mathrm{lmm}), \mathrm{pF}$ & 3.8641 & 9.1004 & 9.1806 & 3.7791 & 9.1525 & 9.3109 & 9.1952 & 9.4916 \\
\hline$G_{12}(1 \mathrm{~mm}), \mathrm{pS}$ & 0 & 0 & 8.5282 & 0 & 0 & 8.9939 & 0 & 8.3603 \\
\hline$C_{12}(2.5 \mathrm{~mm}), \mathrm{pF}$ & 1.6983 & 5.7795 & 5.8019 & 1.7734 & 5.7060 & 5.8289 & 5.7068 & 5.8011 \\
\hline$G_{12}(2.5 \mathrm{~mm}), \mathrm{pS}$ & 0 & 0 & 6.7499 & 0 & 0 & 7.0063 & 0 & 6.2959 \\
\hline$C_{12}(5 \mathrm{~mm}), \mathrm{pF}$ & 1.0081 & 4.1257 & 4.1259 & 1.4478 & 3.9811 & 4.0872 & 3.6349 & 3.7040 \\
\hline$G_{12}(5 \mathrm{~mm}), \mathrm{pS}$ & 0 & 0 & 5.4772 & 0 & 0 & 4.1410 & 0 & 3.3039 \\
\hline \multicolumn{9}{|c|}{ Estimations of one layer parameters } \\
\hline$\varepsilon_{1}(1 \mathrm{~mm})$ & 1.00 & 3.07 & 3.12 & 1.005 & 3.09 & 3.14 & 3.11 & 3.22 \\
\hline$\sigma_{1}(1 \mathrm{~mm})$ & 0 & - & 28.83 & 0 & - & 30.32 & - & 28.26 \\
\hline$\varepsilon_{12}(2.5 \mathrm{~mm})$ & 1.00 & 3.09 & 3.11 & 1.05 & 3.06 & 3.18 & 3.06 & 3.11 \\
\hline$\sigma_{12}(2.5 \mathrm{~mm})$ & 0 & - & 28.50 & 0 & - & 32.95 & - & 26.58 \\
\hline$\varepsilon_{123}(5 \mathrm{~mm})$ & 1.00 & 3.13 & 3.15 & 1.34 & 3.05 & 3.18 & 2.81 & 2.88 \\
\hline$\sigma_{123}(5 \mathrm{~mm})$ & 0 & - & 29.14 & 0 & - & 24.35 & - & 18.04 \\
\hline \multicolumn{9}{|c|}{ Final estimated values of $\varepsilon$ and $\sigma$ for each layer } \\
\hline$\varepsilon_{1}^{m}$ & $1.00(0)$ & $3.07(2.0)$ & $3.12(1.3)$ & $1.005(0.5)$ & $3.09(1.0)$ & $3.14(0.6)$ & $3.11(0.6)$ & $3.22(1.9)$ \\
\hline$\overline{\sigma_{1}^{m}}$ & $0(0)$ & - & $28.83(1.2)$ & $0(0)$ & - & $30.32(3.7)$ & - & $\overline{28.26(3.2)}$ \\
\hline$\varepsilon_{2}^{m}$ & $1.00(0)$ & $3.07(2.0)$ & $3.12(1.3)$ & $2.90(3.6)$ & $3.01(0.0)$ & $3.10(2.9)$ & $2.17(3.3)$ & $2.03(3.3)$ \\
\hline
\end{tabular}

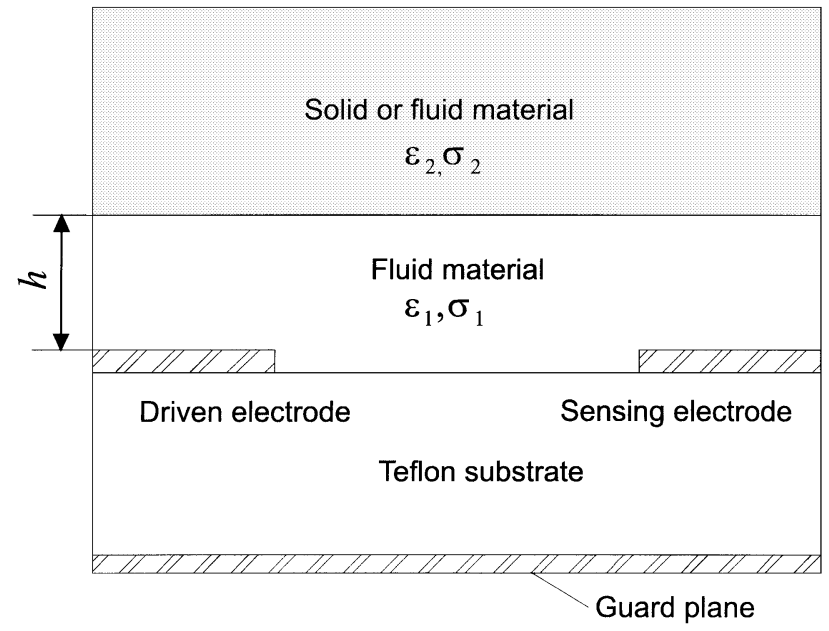

Fig. 9. Generic two-layer test sample with two unknown dielectric layers used in this experiment series.

and the numerical estimates are

$$
\begin{aligned}
& U_{C_{12,1}^{\prime n}} / C_{12,1}^{\prime n} \cong 6.00 \%, \quad U_{C_{12,2}^{\prime n}} / C_{12,2}^{\prime n} \cong 6.00 \%, \\
& U_{C_{12,3}^{\prime n}} / C_{12,3}^{\prime n} \cong 6.00 \%, \quad U_{\varepsilon_{2}} / \varepsilon_{2} \cong 87.60 \%
\end{aligned}
$$

Notice how the uncertainty has grown. The uncertainty of the first layer was $7.2 \%$, then grew to $31.3 \%$ for the second layer, and finally became a huge $87.6 \%$. By using the plots to determine uncertainty, which takes into account the nonlinearity, the regions have uncertainties of $7.6 \%, 51 \%$, and $166 \%$, respectively.

\section{EXPERIMENTAL RESULTS}

The following experimental results have been included to show how the experiment is carried out using a real sensor in the lab. There is no direct connection between the simulated data presented and the experimental data available for use as an example.

\section{A. Description of Setups}

The dielectric properties of insulating materials have been determined experimentally for several cases, all of which can be described by the general diagram in Fig. 9. The fluid material adjacent to the sensor in this series of experiments ensures a perfect contact between the sensor head and the material under test. Since the theoretical analysis of the previous solutions shows that the combination of the $1 \mathrm{~mm}$ and the 5-mm wavelengths is more accurate than other combinations for a two-layer system, this combination is used for the two-layer analysis below.

The measurements and estimates are summarized in Table III. The properties of each layer are summarized at the top part of Table III. In cases 3)-5), we assume that the conductivity of the second layer is known to be negligible. Note that the actual measurements are of capacitance $(\mathrm{pF})$ while the relations described in Figs. 3-5 use capacitance per unit length $(\mathrm{pF} / \mathrm{m})$. The sensor has an effective length of $0.5 \mathrm{~m}$.

Case 1) Sensor in air. This single infinite layer case is used for calibration to account for fringing field effects, manufacturing imperfections, stray lead capacitances, and other sources of small experimental uncertainties. One unknown $\left(\varepsilon_{1}=\varepsilon_{2}=\right.$ $\varepsilon_{0}, \sigma_{1}=\sigma_{2}=0$ ).

Case 2) Sensor in single infinite layer of corn oil. Two unknowns $\left(\varepsilon_{1}=\varepsilon_{2}=\varepsilon, \sigma_{1}=\sigma_{2}=\sigma\right)$. 


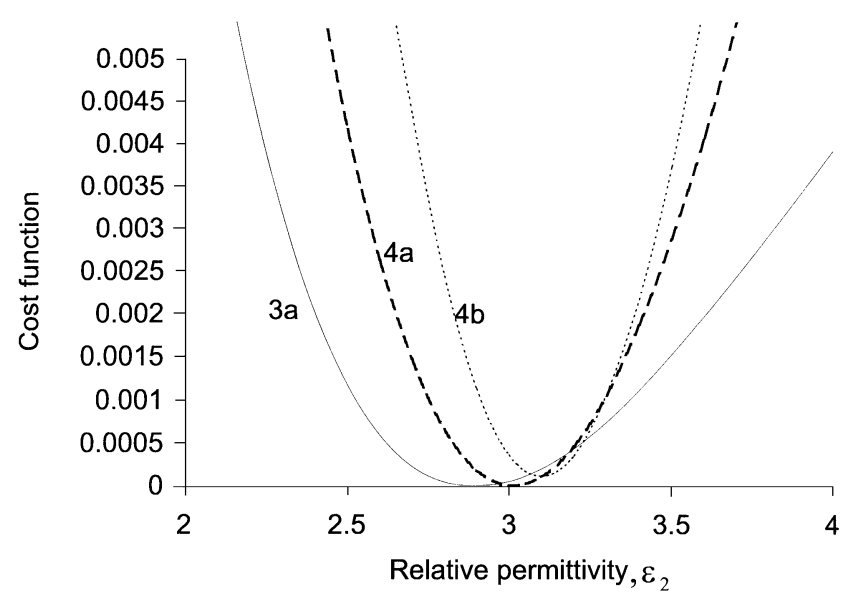

Fig. 10. Cost functions for cases 3a), 4a), and 4b) (Lexan as the second layer).

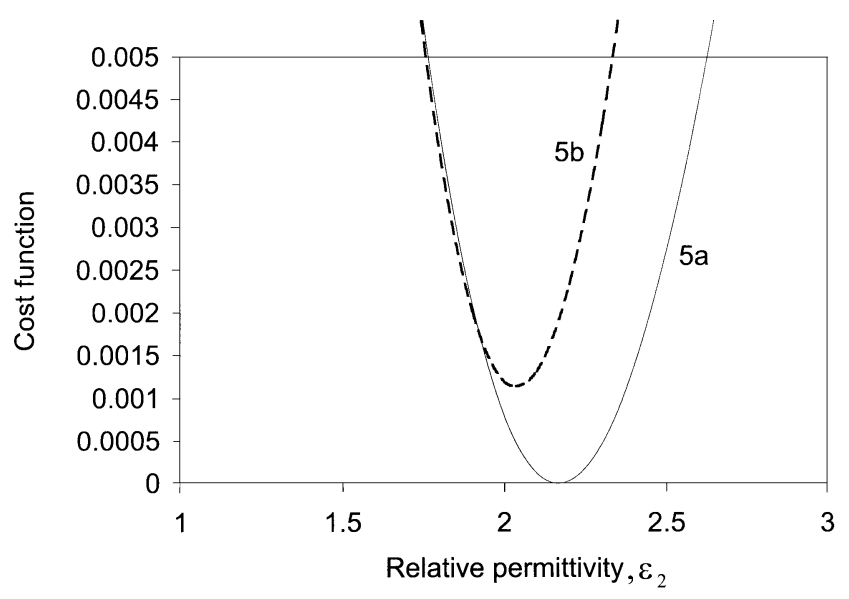

Fig. 11. Cost functions for cases 5a) and 5b) (Teflon as the second layer).

Case 3) First $500 \mu \mathrm{m}$ thick layer-air, second infinite layer-Lexan. Two unknowns $\left(\varepsilon_{1}, \varepsilon_{2}, \sigma_{1}=\sigma_{2}=0\right)$.

Case 4) First $500 \mu \mathrm{m}$ thick layer-corn oil, second infinite layer-Lexan. Three unknowns $\left(\varepsilon_{1}, \varepsilon_{2}, \sigma_{1}, \sigma_{2}=0\right)$.

Case 5) First $500 \mu \mathrm{m}$ thick layer-corn oil, second infinite layer-Teflon. Three unknowns $\left(\varepsilon_{1}, \varepsilon_{2}, \sigma_{1}, \sigma_{2}=0\right)$.

\section{B. Estimation Agorithm}

As a first step, the dielectric permittivity and conductivity of the first layer (fluid layer) is determined from $1 \mathrm{~mm}$ wavelength admittance measurements together with precalculated solution spaces discussed in [13]. This method assumes that the material above the sensor is homogeneous and is of semi-infinite extent. This assumption is approximately valid for the 1-mm wavelength and a first layer thickness of $h=500 \mu \mathrm{m}$.

As a second step, the dielectric permittivity of the second layer is calculated using the 5-mm wavelength data. For cases of dissimilar materials, the conductivity of the second-layer material was zero, $\sigma_{2}=0$. The permittivity of the second layer was found by standard minimization techniques, with the cost function $v$ defined as

$$
v=\sqrt{\left(\frac{G_{12}^{m}-G_{12}^{c}}{G_{12}^{c}}\right)^{2}+\left(\frac{C_{12}^{m}-C_{12}^{c}}{C_{12}^{c}}\right)^{2}}
$$

where $G_{12}^{m}$ and $C_{12}^{m}$ are measured values of transconductance and transcapacitance, and $G_{12}^{c}$ and $C_{12}^{c}$ are the values of transconductance and transcapacitance calculated under the assumption that the first-layer values of dielectric permittivity and conductivity have been determined correctly from the 1-mm wavelength measurements. The cost function is minimized with respect to $\varepsilon$ and $\sigma$ which adjust $C_{12}^{c}$ and $G_{12}^{c}$ in (12).

The cost functions for the cases which require their calculations are shown in Figs. 10 and 11. Fig. 10 shows that the dielectric permittivity of Lexan has been found with a value of the uncertainty of no more than \pm 3.6 percent for $\varepsilon_{2}=3.01$ measured with the guarded parallel-plate cell, and that both the transcapacitance and the transconductance measured and calculated values were in close agreement at the cost function minimum, as indicated by a very low magnitude of the cost function at minimum. The agreement between individual components of transadmittance for measurements with Teflon as the second layer was not as good for the low-frequency measurement point [case 5b)], which is shown in Fig. 11. Nevertheless, the measurement uncertainty did not exceed 3.3 percent for $\varepsilon_{2}=2.1$. The cost function does not go to zero because of uncertainties in the estimation of the dielectric properties of the first layer and measurement uncertainties in the $5 \mathrm{~mm}$ wavelength channel.

\section{CONCLUSION}

The dielectric properties of one and two-layer configurations have been accurately measured using two wavelengths of a three-wavelength interdigital dielectrometry sensor. The focus of this work is on sensitivity analysis and measurement uncertainty evaluation for the stair-step parameter estimation algorithm. It is important to highlight the uncertainty propagation phenomena in order to further improve parameter estimation algorithms and design of fringing field instrumentation. Computer simulations indicate that the measurement uncertainty builds up very quickly as the number of unknowns in the system increases. On the basis of such simulations, the decision can be made whether the electronic and dielectrometry instrumentation is adequate in every specific situation. Future efforts will concentrate on the reduction of the magnitude of disturbance factors and noise sources in dielectrometry measurements and on the development of more sophisticated and generic procedures to characterize the accuracy of fringing electric field sensors.

\section{ACKNOWLEDGMENT}

The authors would like to thank Ansoft Corp. for the donation of Maxwell software.

\section{REFERENCES}

[1] M. Ishido, T. Imaizumi, and M. Toyoda, "Displacement measurement by SAW delay-line oscillator consisting of two $\mathrm{LiNbO}_{3}$ plates with IDT," IEEE Trans. Instrum. Meas., vol. IM-36, pp. 83-86, Mar. 1987.

[2] H. Subramanian, V. V. Varadan, and V. K. Varadan, "Wireless remotely readable microaccelerometer," in Smart Structures Mater.: Smart Electron. MEMS, San Diego, CA, Mar. 1997, pp. 220-228.

[3] I. G. Matis, "On multiparameter control of dielectric properties of laminate polymer materials," in Latvijas PSR Zinatnu Akademijas Vestis, Fizikas un Tehnisko Zinatnu Serija, vol. 6, 1966, pp. 60-67. 
[4] M. C. Zaretsky, L. Mouayad, and J. R. Melcher, "Continuum properties from interdigital electrode dielectrometry," IEEE Trans. Elect. Insul., vol. 23, pp. 897-917, Dec. 1988.

[5] P. A. von Guggenberg and M. C. Zaretsky, "Estimation of one-dimensional complex-permittivity profiles: A feasibility study," J. Electrostat., vol. 34, pp. 263-277, Mar. 1995

[6] S. D. Senturia, N. F. Sheppard, Jr., H. L. Lee, and D. R. Day, "In-situ measurement of the properties of curing systems with microdielectrometry," J. Adhesion, vol. 15, no. 69, pp. 69-90, 1982.

[7] Y. K. Sheiretov and M. Zahn, "Dielectrometry measurements of moisture dynamics in oil-impregnated pressboard," IEEE Trans. Dielect. Elect. Insul., vol. 2, pp. 329-351, June 1995.

[8] A. V. Mamishev, B. C. Lesieutre, and M. Zahn, "Optimization of multiwavelength interdigital dielectrometry instrumentation and algorithms," IEEE Trans. Dielect. Elect. Insul., pp. 408-420, 1998.

[9] V. Shtrauss, D. Pablaks, and A. Kalpinsh, "A multiparameter approach to the test procedure generation for evaluation of the structure of materials," Latvian J. Phys. Tech. Sci., pp. 52-62, 1994.

[10] Y. Du, A. V. Mamishev, B. C. Lesieutre, and M. Zahn, "Measurements of moisture diffusion in transformer pressboard," in IEEE Conf. Elect Insul. Dielect. Phenomena, Atlanta, GA, Oct. 1998, pp. 341-344.

[11] A. V. Mamishev, Y. Du, B. C. Lesieutre, and M. Zahn, "Measuremen of moisture spatial profiles in transformer pressboard," in IEEE Conf. Elect. Insul. Dielect. Phenomena, Atlanta, GA, Oct. 1998, pp. 323-326.

[12] H. W. Coleman and W. G. Steele, Jr., Experimentation and Uncertainty Analysis for Engineers. New York: Wiley, 1989.

[13] A. V. Mamishev, A. R. Takahashi, Y. Du, B. C. Lesieutre, and M. Zahn, "Parameter estimation in dielectrometry measurements," J. Electrostat., vol. 56, pp. 465-492, 1999.

A. V. Mamishev (M'00) received the B.S. degree from the Kiev Polytechnic Institute, Kiev, Ukraine, in 1992, the M.S. degree from Texas A\&M University, College Station, in 1994, and the Ph.D. degree from the Massachusetts Institute of Technology, Cambridge, in 1999, all in electrical engineering.

Currently, he is an Assistant Professor and Director of the Sensors, Energy, and Automation Laboratory (SEAL) in the Department of Electrical Engineering, University of Washington, Seattle. He is an author of more than 40 technical publications. His research interests include sensor design and integration, dielectrometry, electrical insulation diagnostics, and power quality.

Dr. Mamishev serves as a reviewer for the IEEE TRANSACTIONS ON POWER DELIVERY AND IEEE TRANSACTIONS ON DIELECTRICS AND ELECTRICAL INSULATION. He is a recent recipient of the NSF CAREER Award and the Outstanding IEEE Student Branch Counselor Award.

S. R. Cantrell received the B.S. degree in electrical engineering and computer science from the University of California, Berkeley, in 1999, and the M.S. degree in electrical engineering from the University of Washington, Seattle, in 2001. He is currently pursuing the $\mathrm{Ph} . \mathrm{D}$. degree in electrical engineering with a focus in control systems.

His interests include autonomous control, artificial intelligence, and computer graphics.
Y. Du received the B.S. and M.E. degrees from Northwestern Polytechnical University, China, in 1993 and 1994, respectively, and the E.E. and Ph.D. degrees in electrical engineering from the Massachusetts Institute of Technology, Cambridge, in 1999.

Currently, she is a Senior Project Engineer at Underwriters Laboratories (UL), Inc. Prior to join UL, she engaged in professional practice as a Consultant in the analysis and prevention of failures at Exponent Failure Analysis Associates. She has published more than 25 papers in professional conferences and archival journals, including award papers and invited lectures. She is a Registered Professional Engineer in the State of California. Her professional interest and area of expertise include electrical insulation, moisture dynamics in power equipment insulation, failure analysis and prevention of electronics, and electrical equipment.

B. Lesieutre received the B.S., M.S., and Ph.D. degrees in electrical engineering from the University of Illinois, Urbana-Champaign, in 1986, 1988, and 1993, respectively.

From 1993 to 2001, he was Assistant Professor and then Associate Professor of electrical engineering at the Massachusetts Institute of Technology, Cambridge, and a member of its Laboratory for Electromagnetic and Electronic Systems. He is presently Visiting Associate Professor at the School of Electrical and Computer Engineering at Cornell University, Ithaca, NY

M. Zahn received the B.S.E.E., M.S.E.E., Elect. Eng., and Sc.D. degrees, al from the Department of Electrical Engineering, Massachusetts Institute of Technology (MIT), Cambridge.

From 1970 to 1980 , he was a Professor of electrical engineering at the University of Florida, Gainesville. He joined the MIT faculty in 1980 and is now Professor of electrical engineering in the Laboratory for Electromagnetic and Electronic Systems and in the MIT High Voltage Research Laboratory. He is also the Director of the MIT Course VI-A Electrical Engineering and Computer Science Internship Program, a cooperative work/study program with industry and the Government. His fields of research and interest include electrooptical field and charge mapping measurements; high-voltage charge transport and breakdown phenomena in dielectrics; flow electrification phenomena in electric power apparatus; development of capacitive and inductive sensors of dielectric, conduction, and magnetic properties of media as well as related physical properties; electrohydrodynamic and electrokinetic interactions with charged and polarizable fluids; ferrohydrodynamic interactions with magnetizable fluids; continuum electromechanics of electrofluidized and magnetically stabilized beds; magnetic diffusion phenomena and forces in MAGLEV systems; and magnetic field devices for micro and nanoelectromechanical system (MEMS/NEMS) applications. 